\title{
Cryogenic Hydrogen Oxygen Propulsion System (CHOPS) for Planetary Science Missions
}

\author{
Shuvo Mustafi ${ }^{1}$, Conor A. Nixon ${ }^{1}$, Noah Petro ${ }^{1}$, Xiaoyi Li ${ }^{1}$, Lloyd R. Purves ${ }^{1}$, \\ Alber Douglawi², Steven P. Simpson ${ }^{2}$, Ali Hedayat ${ }^{2}$
}

\begin{abstract}
${ }^{1}$ NASA Goddard Space Flight Center (GSFC), Greenbelt, MD 20771
${ }^{2}$ NASA Marshall Space Flight Center (MSFC), Huntsville, AL 35812
\end{abstract}

Point of Contact:

shuvo.mustafi@nasa.gov

+1 301-286-7436 


\subsection{Executive Summary}

In this white paper we examine a new type of propulsion system for planetary science missions: a cryogenic hydrogen oxygen propulsion system (CHOPS). Despite the considerable advantages of CHOPS over other chemical propulsion systems cryogenic rocket engines have mostly been used for the launch phase of missions due to the perceived challenges of long-duration in space storage of cryogenic propellants. We show that new developments in passive cryogen storage technology can solve this problem, which now renders CHOPS viable for in-space propulsion. CHOPS engines offer a significant specific impulse $\left(I_{\mathrm{sp}}\right)$ advantage over traditional hypergolic engines, thereby reducing the launched mass of planetary science spacecraft. CHOPS also offers several additional advantages over traditional hypergolic engines that are especially significant for planetary science missions, and in particular for landers at sites of astrobiological interest. These include 'clean' burning exhaust, similar to a fuel cell, which produces only water; throttleable landings; the possibility to use the propellant to generate electricity, allowing for longer lived missions than missions using just a primary battery; and the possibility to use the fuel as radiation shield. We recommend that cryogenic hydrogen oxygen propulsion systems, including those already under development at MSFC, GSFC, and elsewhere, are evaluated for planetary applications in ground testing, leading to an in-space demonstration.

\subsection{Background}

In-space propulsion is necessary for spacecraft to change course, unload (debias) reaction wheels, enter orbit, dock, and land on solar system targets. In-space propulsion technologies are summarized in Error! Reference source not found..

\begin{tabular}{|c|c|c|c|c|c|}
\hline & $\begin{array}{c}\text { Specific } \\
\text { Impulse (s) }\end{array}$ & $\begin{array}{c}\text { TRL } \\
\text { Hypergolic (e.g. MMH+NTO) }\end{array}$ & $\begin{array}{c}\text { Maximum } \\
\text { AU }\end{array}$ & $\begin{array}{c}\text { 'Clean' for } \\
\text { astrobiology }\end{array}$ & $\begin{array}{c}\text { Dual-use } \\
\text { for power } \\
\text { generation }\end{array}$ \\
\hline Hylar Electric Propulsion (SEP) & $500-4500$ & 9 & 5.2 & $\mathrm{~N}$ & $\mathrm{~N}$ \\
\hline $\begin{array}{c}\text { Nuclear Electric Propulsion (NEP), } \\
\text { Nuclear Thermal Propulsion (NTP) }\end{array}$ & $\begin{array}{c}\text { NEP: } 8000+, \\
\text { NTP: } 850-1000\end{array}$ & $4-5$ & Any & $\mathrm{N}$ & $\mathrm{Y}$ \\
\hline $\begin{array}{c}\text { Cryogenic Hydrogen Oxygen } \\
\text { Propulsion System (CHOPS) }\end{array}$ & $440+$ & $4-5$ & Any & $\mathrm{Y}$ & $\mathrm{Y}$ \\
\hline
\end{tabular}

\section{Table 1: Comparison of in-space propulsion technologies}

Hypergolic engines: Currently, the most commonly used in-space propulsion is 'hypergolic' propulsion, where room temperature liquid fuel and oxidizer, e.g. mono-methyl hydrazine (MMH) and nitrogen tetraoxide (NTO), are burned in a chemical rocket engine. Through many decades of use, this technology has achieved high technology readiness level (TRL) for flight, but nonetheless has some drawbacks, including a low specific impulse $\left(\mathrm{I}_{\mathrm{sp}}\right)$, toxicity, and an exhaust composed of organic (CHON) molecules which is contaminating for astrobiological sites, making it less attractive for astrobiology applications such as landers for ocean worlds or Mars.

Solar-electric propulsion (SEP): SEP has been used in missions where low-thrust over long periods are acceptable. This type of technology is cleaner than hypergolic engines in the astrobiological sense, emitting only (typically) xenon atoms, but is unsuitable for high thrust 
applications such as orbit insertion or landing, and the required solar panels become impractically large at locations that are distant from the sun.

Nuclear electric propulsion (NEP) and Nuclear thermal propulsion (NTP): NEPs use nuclear fission reactors to generate electricity for ion/plasma engines. NTPs use the thermal energy from nuclear fission reactors for propulsion. Neither of these nuclear propulsion technologies have been proven in space for planetary science missions. Neither of them scale well for small planetary science applications. In addition, the radioactive energy sources for both these propulsion technologies invoke additional planetary protection concerns, especially for landers.

Cryogenic Hydrogen Oxygen Propulsion System(CHOPS): Liquid hydrogen (LH2) and liquid oxygen (LO2) cryogenic propellants can dramatically enhance NASA's ability to explore the solar system due to their superior specific impulse $\left(\mathrm{I}_{\mathrm{sp}}\right)$ capability. Launch vehicles have used these and other cryogenic propellants for decades. Historically, the challenge of storing cryogenic propellants for long durations has resulted in cryogenic propulsion remaining undeveloped for planetary science missions. However, with the advent of new propulsion and cryogenic storage technologies NASA's GSFC and MSFC have formed a partnership to develop a Cryogenic Hydrogen Oxygen Propulsion System (CHOPS) for planetary science missions that would be cleaner, safer and provide significant mass advantages over traditional hypergolic propulsion systems. CHOPS would allow NASA to deliver more payloads to targets of interest, launch on smaller and less expensive launch vehicles, or both, thereby enabling a large variety of planetary science missions. CHOPS propelled missions would also reduce the risk for planetary contamination, provide a mass efficient power alternative to batteries and provide radiation shielding for sensitive spacecraft electronics and instruments.

\subsection{New Technology 1: CHOPS Engine}

A CHOPS engine would enable a variety of missions. However, these missions present a unique and challenging set of requirements for the propulsion system. The engine would need to be capable of thousands of seconds of burn time, multiple starts, and long dwells in between each engine burn. An example CHOPS engine is depicted in Figure 1.

The engine design uses pump fed propellants. Traditional pump fed rocket engines utilize gas-powered turbines to produce power for each pump. With the required small flow rate for a CHOPS engine, these types of turbines would be nearly impossible to manufacture with current technology. Instead, a brushless DC motor would run each pump with electrical power supplied by on-board batteries. However, the specifications required for such motors are achievable based on experience in developing similar systems at MSFC. Motors that operate at LH2 temperatures have been demonstrated in industry and may benefit by taking advantage of the cold environment to increase power density. Since the required burn time of CHOPS engines may be in the thousands of seconds, chamber life is another challenging requirement. A dual cooling circuit could be implemented where LH2 would cool the throat and chamber section, while LO2 
would cool part of the nozzle. A carbon-carbon nozzle extension would complete the expansion ratio out to 128:1. Active cooling circuits at higher pressure would allow for autogenous repressurization of the cryogenic propellant tanks and eliminate the need for extra helium pressurization gas. For engine starts, the propellant would be allowed to bleed through the active cooling circuits and that chill down propellant would be used for settling of the tank and early pressurization. Auxillary heaters could also be used for pre-engine start pressurization. Gaseous hydrogen and gaseous oxygen RCS thrusters can be used to save mass where high pressure from pump discharge could refill RCS supply tanks for use during planetary missions.

\subsection{New Technology 2: CHOPS Passive Cryogen Storage System (PCSS)}

Cryogens are usually stored at temperatures that are significantly lower than their surroundings. As a result they are almost always heat sinks that absorb heat. This heat can vaporize the cryogen, thereby losing the very commodity that is being stored. The loss of cryogen in space can lead to mission reduction or mission loss. The CHOPS Passive Cryogen Storage System (PCSS) enables long term in-space storage of cryogens. Cryogens are stored both actively (using powered cooling and heat extraction devices such as cryocoolers and refrigerators) and passively (using unpowered passive thermal isolation systems). The Passive Cryogen Storage System (PCSS) technology uses several advanced thermal isolation systems to reduce the heat incident on the cryogen storage system. High performing active cryogen storage systems can alsoimplement the PCSS strategies as these decrease the amount of heat that the active cooling devices need to extract. Depending on the misssion duration, PCSS can reduce or even eliminate the mass and power penalties that spacecraft with active cooling devices face.

PCSS consists of a range of thermally insulating components that by themselves or in combination with each other or other storage strategies, such as cryocoolers or cryogen subcooling, can reduce or eliminate vented cryogenic propellant loss. There are three potential paths for heat transfer into a cryogen: conduction, convection and radiation. PCSS components address each of these. Conduction may be reduced using low thermal conductivity and high strength composite materials such as T300. Convection may be reduced using a light weight vacuum jacket such as Quest Thermal Group's (QTG's) Load Responsive MLI (LRMLI). Radiation may be reduced by using mass and thermally efficient radiation shields such as QTG's Integrated MLI (IMLI) and sun shields. Depending on the mission, these components would have to be strategically implemented to minimize the heat leak into the cryogen while simultaneously allowing passive heat extraction from the cryogen tank by radiating heat to deep space.

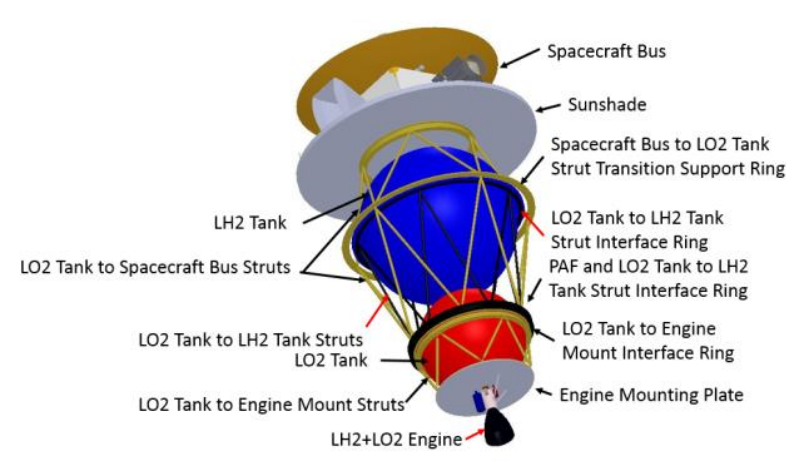

(a)

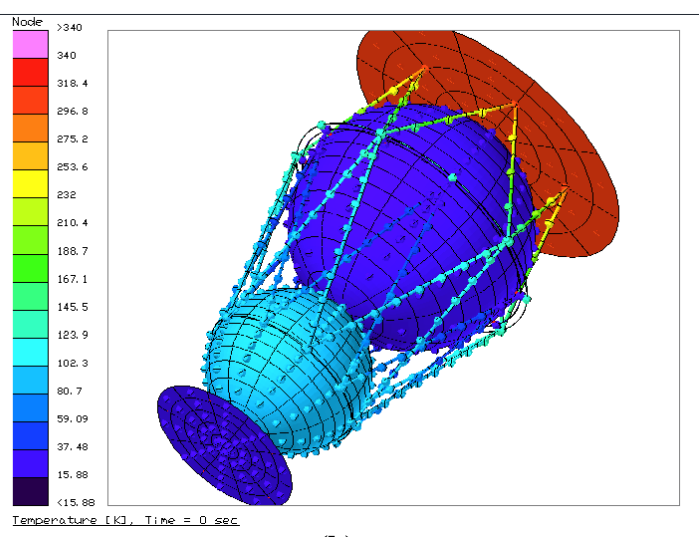

(b)

Figure 2: (a) CHOPS for a representative planetary science spacecraft (b) Thermal analysis of PCSS for the same spacecraft. 


\begin{tabular}{|c|c|c|}
\hline Example Planetary Science Mission Parameters & CHOPS & MMH+NTO \\
\hline Total $\Delta$ V [m/s] & 5887 & 5887 \\
\hline Main Propulsion Mission Duration [Years] & 8.5 & 8.5 \\
\hline Non Main Propulsion Dry Mass [kg] & 595 & 595 \\
\hline Dry Mass with 25\% Dry Mass Contingency [kg] & 880 & 1053 \\
\hline Launch Mass with 25\% Dry Mass Contingency [kg] & 3174 & 5587 \\
\hline Launched Spacecraft Fuel Mass (LH2 Vs. MMH) [kg] & 436 & 1744 \\
\hline Launched Spacecraft Oxidizer Mass (LO2 Vs. NTO) [kg] & 1964 & 2790 \\
\hline
\end{tabular}

Table 2: Comparison of CHOPS and MMH+NTO propelled representative spacecraft.

Figure 2(a) shows a view of the CHOPS PCSS and the CHOPS engine that is used for a representative planetary science spacecraft [1]. The structural supports on the spacecraft are provided using low thermal conductivity composite T300/RS-3C struts, sized to balance both structural, launch and thermal requirements. This arrangement of struts allows for the lower temperature LH2 tank, with a normal boiling point (NBP) of $20 \mathrm{~K}$ and subcooled to $16 \mathrm{~K}$, to be conductively isolated from both the launch interface and the spacecraft bus which may be at temperatures above $300 \mathrm{~K}$. The LO2 tank, with a NBP of $90 \mathrm{~K}$, further isolates the lower temperature LH2 tank from the $300 \mathrm{~K}$ interface.

A sunshade separates the warmer spacecraft bus side of the spacecraft from the colder propulsion side. The spacecraft will normally point such that the bus is facing the sun. However, the thermal design of the tanks allows tolerance of Sun views and increased heat input during short durations for engine burn and communication maneuvers. This arrangement also allows sufficient view to space for both the LO2 tank and the LH2 tank. Depending on the mission, the view to space for the tanks can be sufficient such that the heat leak into the LO2 tank is considerably diminished, eliminated, or even negated causing a tank chill down, and the heat leak into the LH2 tank can also be considerably diminished. To further minimize LH2 loss by phase-change, the LH2 could be subcooled on the launch pad to $16 \mathrm{~K}$. [2, 3, 4, 5]

The conductive isolation for the $\mathrm{LH} 2$ and $\mathrm{LO} 2$ tanks on the spacecraft are provided by the T300/RS-3C struts. The arrangement of the struts as described above reduces the conductive load on both tanks. The convective isolation on the tanks while it is on the ground is provided using a 5 layer Load Responsive MLI (LRMLI).The radiative isolation on the tank is provided using 40 layers of Integrated MLI (IMLI). IMLI and LRMLI are produced by Quest Thermal Group. [6, 7] The performance of the LRMLI and IMLI was scaled from demonstrated performance. [8,9]

The thermal analysis of the cryogenic fluid management for this representative spacecraft is performed using Thermal Desktop (TD) software. Figure 2(b) shows the TD analysis performed for the spacecraft in deep space. The analysis indicates that the LO2 tank loses about $42 \mathrm{~mW}$ of net heat if the tanks are pointed toward deep space. As a result, the oxygen can actually de-energize and densify during missions where the cryogenic tanks can be pointed towards deep space for long durations, so there is no loss in oxygen propellant due to phase change and this is accomplished without any active cooling. The in-space TD analysis also indicates that the LH2 tank has a nominal heat gain of $71 \mathrm{~mW}$ in deep space. For a representative CHOPS mission lasting 8.5 years this heating of the subcooled LH2 tank would result in about $44 \mathrm{~kg}$ of LH2 loss to phase change. 
Table 2 compares the representative CHOPS Mission with the same mission if it was propelled using traditional hypergolic MMH+NTO propulsion. It shows a 43\% launched mass savings for a spacecraft propelled with CHOPS when compared to another spacecraft with the same nonpropulsion dry mass conducting the same mission but being propelled by MMH+NTO. This launched mass savings can allow the CHOPS propelled spacecraft to launch on a smaller and less expensive launch vehicles. This whitepaper presents the use of CHOPS for a representative planetary science mission in significant detail. However, CHOPS can be adopted for a variety of other missions and in many cases CHOPS could be mission enabling given the significant reduction in launched mass provided by the high specific impulse of hydrogen-oxygen propulsion systems. Table 3 shows the summary of a parametric study on the launch mass savings for using CHOPS instead of the traditional MMH+NTO propulsion system for planetary science missions for a range of desired non-main propulsion dry masses and a range of desired mission $\Delta$ Vs.

Given the significant amount of mass savings that is achievable for a range of dry masses and $\triangle \mathrm{Vs}, \mathrm{CHOPS}$ is also being investigated for other types of planetary science missions including orbiters, landers, and sample return missions. While the high specific impulse that CHOPS provides is very attractive for a variety of missions, there are also several other features that make CHOPS particularly appealing for planetary science missions. The only effluent byproduct from the CHOPS engine is water. CHOPS would reduce the possibility of landers and sample return missions contaminating the planetary bodies that they are investigating. If hydrogen and oxygen is being carried for propulsion, these propellants can also be used as reactants for fuel cells thus providing a mass efficient alternative power source to large batteries. Hydrogen is also the most mass efficient material radiation shield. An appropriately sized and positioned LH2 tank can provide effective radiation shielding for sensitive electronics and instruments on orbiters, landers or sample return vehicles in high radiation environments [10].

\begin{tabular}{|c|c|c|c|}
\hline \multicolumn{4}{|c|}{ Parametric Launch Mass Savings for using CHOPS Vs MMH+NTO [kg] } \\
\hline & \multicolumn{3}{|c|}{ Mission $\mathbf{\Delta V}[\mathrm{m} / \mathrm{s}]$} \\
\hline $\begin{array}{c}\text { Non-Main Propulsion } \\
\text { Dry Mass [kg] }\end{array}$ & $\mathbf{2 5 0 0}$ & $\mathbf{5 0 0 0}$ & $\mathbf{7 5 0 0}$ \\
\hline $\mathbf{2 5 0}$ & 118 & 843 & 3,664 \\
\hline $\mathbf{5 0 0}$ & 255 & 1,469 & 6,251 \\
\hline $\mathbf{1 0 0 0}$ & 529 & 2,721 & 11,425 \\
\hline
\end{tabular}

Table 3: Launch mass savings by using CHOPS instead of a traditional MMH+NTO propulsion.

\subsection{Representative Science Applications}

\subsection{Lunar Lander Missions:}

The Lunar Any Site Sampler Orbiter (LASSO) mission enables repeated sample return from any desired site from the surface of Earth's moon. This mission is enabled by the use of CHOPS. LASSO consists of a lunar lander and a propellant depot. The lunar lander with a CHOPS engine and a propellant storage system leaves the propellant depot and lands at any desired lunar location. The lunar lander collects lunar samples, returns to the depot in lunar orbit, deposits the sample and refills the cryogenic propellant tank. The lunar lander can land 3 to 4 times on any location on the lunar surface. The mission architecture is also designed so that the propellant depot can be refilled 
from regular servicing missions that are planned for the Deep Space Gateway (DSG) at the Near Rectilinear Halo Orbit (NRHO) around the moon. In the presence of a serviced DSG, LASSO can pick up samples from as many lunar surface sites and as many times as desired. LASSO's ability to access any location on the lunar surface will not only allow important science, it can also serve as a precursor to characterize landing sites in support of human missions.

\subsection{Landers for Ocean Worlds \\ CHOPS offers} multiple potential benefits for landers to icy moon/ocean worlds destinations that may harbor life, and are high priority targets for astrobiology. As an example, the recent study for a Europa Lander reference mission [11] envisages a large, multielement mission (Figure 3) consisting of a cruise stage (the Carrier Relay Orbiter), De-orbit Stage, Descent Stage and Lander. The multiple elements add up to a heavy vehicle stack: 15 tons (wet) at launch, which can only be

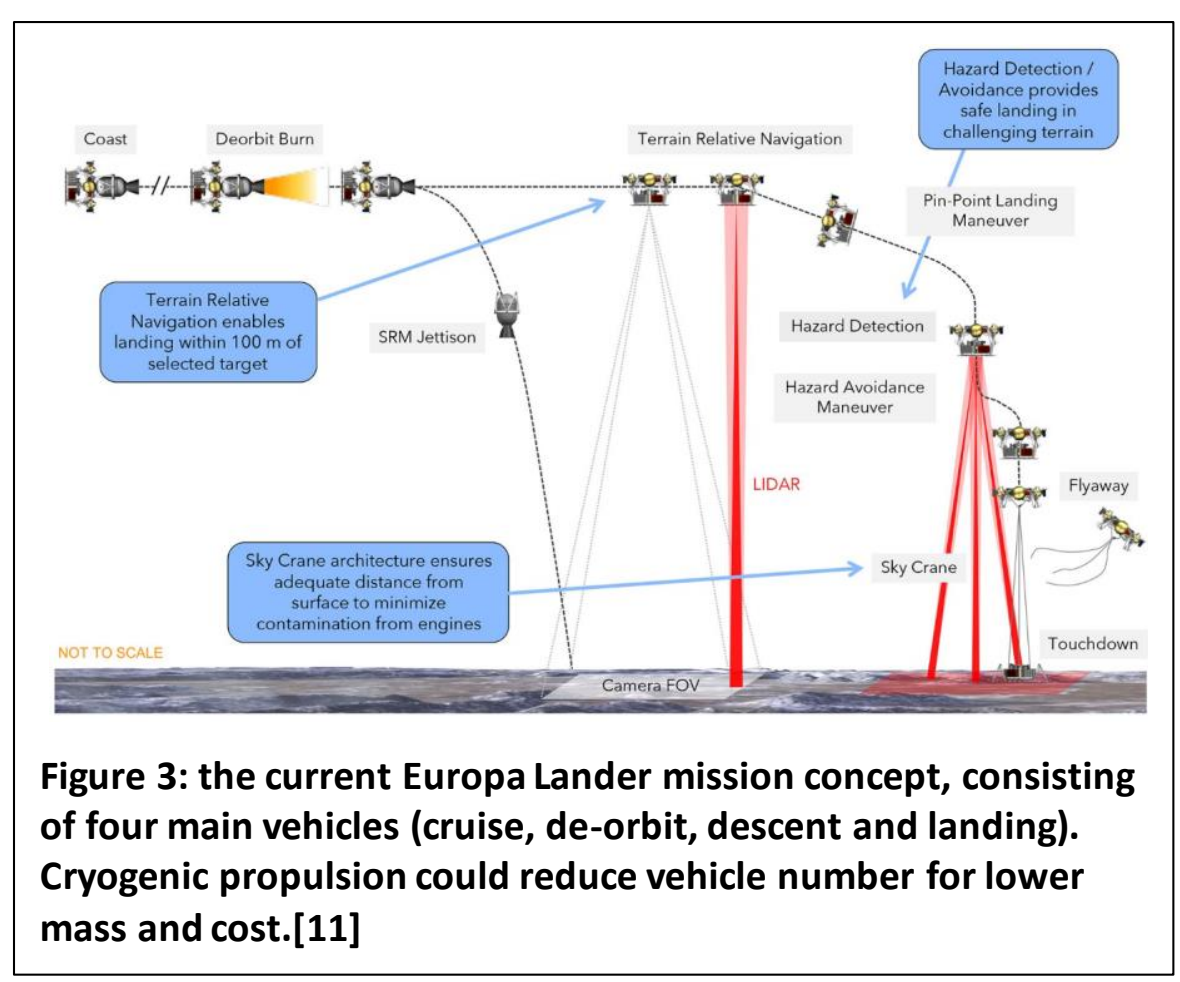
launched by an SLS Block 1B rocket, currently still under development. The mass problem is exacerbated by the relatively low $\mathrm{I}_{\mathrm{sp}} \mathrm{MMH}+\mathrm{NTO}$ propellants, significant amounts of which are needed for Jovian orbit insertion, Europa de-orbit and final landing. Five main advantages accrue from using CHOPS for this mission:

(1) Lower spacecraft wet mass at launch due to the higher $\mathrm{I}_{\mathrm{sp}} \mathrm{LH} 2+\mathrm{LO} 2$ propellants, opening the possibility of lower-cost, existing launch vehicles (e.g. Falcon Heavy, Delta-IV Heavy) than the SLS Block 1B.

(2) Clean-burning exhaust (water rather than carbon and nitrogen combustion products) leading to lower landing site contamination, improving science and planetary protection.

(3) The possibility of generating lander electrical power through fuel-cells, increasing available power beyond the current mission concept battery power or reducing battery mass.

(4) The possibility of combining or eliminating mission elements to reduce mass further. e.g. combining de-orbit and descent stages or descent and landing stages, more similar to Apollo, enabled by use of higher $\mathrm{I}_{\mathrm{sp}}$ and contamination-free exhaust propellant.

(5) The possibility of using the LH2 fuel as a radiation shield in the high radiation Jupiter environment.

The Europa Lander mission has been used as an example in this whitepaper since the reference study is in the public domain. However, this technology can equally be applied to future landers and orbiters for Enceladus, Europa, Triton, Ceres etc, or Mars polar regions. 


\subsection{Conclusions and Recommendations}

This paper finds that:

- Propulsion: The significant amounts of mass savings of CHOPS propelled planetary science spacecraft compared to traditional $\mathrm{MMH}+\mathrm{NTO}$ propelled spacecraft would allow $\underline{\mathrm{CHOPS}}$ propelled spacecraft to carry out missions that would otherwise not 'close' using existing launch vehicles, or enable these spacecraft to launch on cheaper launch vehicles, or both.

- Fuel storage: The PCSS design allows for passive storage of the LH2 and LO2 propellants for mission durations that can extend beyond a decade with no LO2 loss and only a small vaporized $\underline{\mathrm{LH} 2}$ loss (accounting for which still provides many tons of launched mass savings over a $\mathrm{MMH}+\mathrm{NTO}$ propelled spacecraft conducting the same mission).

- Missions Enabled: A variety of missions that require high $\Delta \mathrm{V}$ maneuvers would benefit from the use of CHOPS. Missions that are too far from the Sun for solar electric propulsion or missions that are destined for planetary bodies without atmospheres for aero-braking or missions that have prohibitively high $\Delta \mathrm{V}$ requirements because of the lack of planetary alignments for gravity assists can be enabled by CHOPS.

- Planetary Protection: CHOPS provides a lower risk for planetary contamination than traditional hypergolic propulsion. CHOPS can potentially offer a mass efficient alternative to batteries for a spacecraft power source. CHOPS also has the potential for providing mass efficient radiation shielding to sensitive electronics and instruments.

We recommend:

- Ground test: a technology development initiative between SMD and STMD to develop and test CHOPS technology on the ground, leading to:

- In-space test: an in-space test flight of the CHOPS technology including in-space engine restarts, and evaluation of the PCSS propellant storage during a long-duration test flight.

\subsection{References}

[1] Mustafi, S., et al, "Cryogenic propulsion for the Titan Orbiter Polar Surveyor (TOPS) Mission", Cryogenics, 74, 81-87, 2016. [2] Mustafi, S., et al, "Subcooling Cryogenic Propellants for Long Duration Space Exploration," AIAA2009-6584. [3] Mustafi, S., et al, "Subcooling for Long Duration In-Space Cryogenic Propellant Storage," AIAA2010-8869. [4] Naes, L., et al, "WISE Solid Hydrogen Cryostat Design Overview," SPIE Conference Proceedings Vol. 5904, 2005. [5] Creare, "20K - 20 W Cryocooler System Scaling Study," NASA Scaling Study - Final Report, Contract NNG12LN29P, 2012. [6] Dye, S., et al, "Integrated and Load Responsive Multilayer Insulation," Advances in Cryogenic Engineering 55, 946-953, 2010. [7] Dye, S., et al, "Novel load responsive multilayer insulation with high in-atmosphere and on-orbit thermal performance," Cryogenics 52, 243-247, 2012. [8] Quest Thermal Group, "Load Responsive Multi-Layer Insulation: Thermal Insulation with High In-atmosphere and On-Orbit Performance," NASA SBIR Phase 2 Final Report, Contract NNX10CA70C, 2012. [9] Quest Thermal Group, "Integrated Multi-Layer Insulation," NASA SBIR Phase 2 Final Report, Contract NNCO8CA13C, 2010. [10] Li, X., et al, "Cryogenic Hydrogen Radiation Shield for Human Spaceflight," Patent US 10,144,535 B2, 2018. [11] Hand, K. P., et al., "Europa Lander Study 2016 Report: Europa Lander Mission," JPL Document 97667. 